\title{
ON LEFT AMENABLE SEMIGROUPS WHICH ADMIT COUNTABLE LEFT INVARIANT MEANS
}

BY E. GRANIRER

Communicated by M. M. Day, July 12, 1962

Introduction. This work is closely connected with [5] and we shall freely use the notations of $[5, \S 2]$.

Let $G$ be a semigroup which admits countable left invariant means (i.e., $M l(G) \cap Q l_{1}(G) \neq \varnothing$, where $M l(G)$ is the set of left invariant means) and let $g l(G)=\left\{\phi ; \phi \in m(G)^{*}, L_{\diamond} \phi=\phi\right.$ for $\left.g \in G\right\}$. By Theorem 4.2 of [5] $G$ contains finite groups which are left ideals with left cancellation, i.e. by $[5, \S 2]$ (1.i.l.c.). ${ }^{1}$ Let $\left\{A_{\alpha}\right\}_{\alpha \in I}$ be the set of all finite groups which are (1.i.l.c.) in $G$ and define for $\alpha, \beta \in I, \alpha \cdot \beta=\beta$. The indices set $I$ becomes thus a semigroup with semigroup algegra $l_{1}(I)$ and second conjugate algebra $m(I)^{*}$ (as defined in Day [3, p. 526]). As proved in Theorem 1 of M. M. Day [3, p. 530] $g l(G)$ is also a subalgebra of $m(G) *$ (when regarded as the second conjugate algebra of $l_{1}(G)$ ).

A linear positive isometry from $m(I)^{*}$ onto $g l(G)$ which displays the inner structure of $g l(G)$ is constructed in this paper. This isometry is also an algebraic isomorphism from the algebra $m(I)^{*}$ onto the algebra $\operatorname{gl}(G)$.

$A=\mathrm{U}_{\alpha \in I} A_{\alpha}$ is a right minimal ideal (this is the result of Clifford [1,] for proof see [5, Lemma 3.1 and Remark 3.1]) and moreover, the $A_{\alpha}$ 's as finite groups are isomorphic to one another (see [6] end of proof of Theorem E) therefore the number $N$ of elements of $A_{\alpha}$ does not depend on $\alpha$. We now define the linear operator $T: m(G) \rightarrow m(I)$ :

$$
\text { for } \alpha \in I \quad(T f)(\alpha)=\frac{1}{N} \sum_{o \in A_{\alpha}} f(g) .
$$

This operator has the following properties:

(1) If $f(g) \geqq 0$ for each $g$ in $G$ then $(T f)(\alpha) \geqq 0$ for each $\alpha \in I$ (obvious).

(2) $T 1_{G}=1_{I}$ (obvious).

(3) $T\left(l_{a} f\right)=T(f)$ for each $a$ in $G$ and $f$ in $m(G)$ :

$\left(T l_{a} f\right)(\alpha)=\frac{1}{N} \sum_{g \in A_{\alpha}}\left(l_{a} f\right)(g)=\frac{1}{N} \sum_{g \in A_{\alpha}} f(a g)=\frac{1}{N} \sum_{o \in A_{\alpha}} f(g)=(T f)(\alpha)$

( $a A_{\alpha}=A_{\alpha}$ since $A_{\alpha}$ is a finite (1.i.l.c.))

${ }^{1}$ A finite group $B C G$ is a (1.i.1.c.) if $g B=B$ for each $g$ in $G$. 
(4) $T$ is linear and $\|T f\| \leqq\|f\|$ for $f$ in $m(G)$. Linearity is evident and

$$
\begin{aligned}
\|T f\| & =\sup _{\alpha \in I}|(T f)(\alpha)|=\sup _{\alpha \in I} \frac{1}{N}\left|\sum_{g \in A_{\alpha}} f(g)\right| \leqq \sup _{\alpha \in I} \frac{1}{N} \sum_{o \in A_{\alpha}}|f(g)| \\
& \leqq \frac{1}{N} \sum_{g \in A_{\alpha}}\|f\|=\|f\| .
\end{aligned}
$$

(5) $T[m(G)]=m(I)$ since if $h$ is in $m(I)$ then we define $f$ in $m(G)$ as follows: for $g \in A_{\alpha}$ let $f(g)=h(\alpha)$ and this for each $\alpha \in I$, for $g$ not in $A=\mathrm{U}_{\alpha \in I} A_{\alpha}$ (if there exist at all such g's) let $f(g)=0$. Obviously $f$ is in $m(G)$ and:

$$
(T f)(\alpha)=\frac{1}{N} \sum_{g \in A_{\alpha}} f(g)=\frac{1}{N} \sum_{g \in A_{\alpha}} h(\alpha)=h(\alpha) .
$$

Thus $T f=h$, but moreover the above chosen $f$ satisfies also $\|f\| \leqq\|h\|$. Thus the image of the closed unit ball in $m(G)$ by $T$, is the whole closed unit ball of $m(I)$.

(6) If $B C G$ is a finite set then $\left(T 1_{B}\right)(\alpha)$ does not vanish at most on a finite subset of $I$. In fact $\left(T 1_{B}\right)(\alpha)=(1 / N) \sum_{g \in A_{\alpha}} 1_{B}(g)$, thus $T\left(1_{B}\right)$ does not vanish only for those $\alpha$ which satisfy $B \cap A_{\alpha} \neq \varnothing$. Since $B$ is finite there is at most a finite number of such $\alpha$.

If $S$ is a set then let $c_{0}(S)^{\perp} C m(S) *$ be defined by

$$
c_{0}(S) \perp=\left\{\phi ; \phi\left(1_{g}\right)=0 \text { for each } g \text { in } S\right\}
$$

We are now ready to prove the following:

Theorem. $T^{*}: m(I)^{*} \rightarrow m(G)^{*}$ is a linear positive isometry from $m(I)^{*}$ onto $g l(G)$ such that $T^{*}\left[Q l_{1}(I)\right]=Q l_{1}(G) \cap g l(G)$ and

$$
T^{*}\left[c_{0}(I)^{\perp}\right]=c_{0}(G)^{\perp} \cap \operatorname{gl}(G) .
$$

Proof. $\left(T^{*} \phi\right) f=\phi(T f)$ for $\phi$ in $m(I)^{*}$ and $f$ in $m(G) . T^{*}$ is linear and moreover is isometric since:

$$
\begin{aligned}
\left\|T^{*} \phi\right\| & =\sup _{f \in m(G),\|f\| \leqq 1}\left|\left(T^{*} \phi\right)(f)\right|=\sup _{f \in m(G),\|f\| \leqq 1}|\phi(T f)| \\
& \left.={ }^{*}\right) \sup _{h \in m(I),\|n\| \geqq 1}|\phi(h)|=\|\phi\|
\end{aligned}
$$

(for $\left(^{*}\right)$ see (5) above). Now for $\phi$ in $m(I)^{*}, f$ in $m(G)$ and $a$ in $G$

$$
\left(T^{*} \phi\right)\left(l_{a} f\right)=\phi\left(T l_{a} f\right)=\phi(T f)=\left(T^{*} \phi\right)(f)
$$

(see (3) above) which implies that $T^{*}\left(m(I)^{*}\right) \subset g l(G)$. We prove now that $T^{*}\left(m(I)^{*}\right)=g l(G)$. In order to do this we have to prove at first 
that if $\phi$ is in $g l(G)$ and $f$ in $m(G)$ is such that $T f=0$ (i.e., $T f(\alpha)$ $=(1 / N) \sum_{g \in A_{\alpha}} f(g)=0$ for each $\left.\alpha \in I\right)$ then $\phi(f)=0$. In fact if $G-A$ will denote those elements of $G$ which are not in $A=\bigcup_{\alpha \in I} A_{\alpha}$ (this may be an empty set) and if $a$ is some element of $A$, then for any $f^{\prime}$ of $m(G)$ and $g$ of $G$ we have

$$
\left(l_{a}\left(f^{\prime} 1_{G-A}\right)\right)(g)=\left(f^{\prime} 1_{G-A}\right)(a g)=f^{\prime}(a g) 1_{G-A}(a g)=0
$$

since $a g$ belongs to $A$ for any $g$ of $G$ (see remark (3.1) of [5]). Thus:

$$
\phi\left(f^{\prime}\right)=\phi\left(f^{\prime} 1_{A}\right)+\phi\left(f^{\prime} 1_{G-A}\right)=\phi\left(f^{\prime} 1_{A}\right)+\phi\left(l_{a}\left(f^{\prime} 1_{G-A}\right)\right)=\phi\left(f^{\prime} 1_{A}\right) .
$$

Let us pick some $\alpha_{0}$ of $I$ and let $a_{1}, \cdots, a_{N}$ be the $N$ elements of $A_{\alpha_{0}}$. If $a$ is an arbitrary but fixed element of $A$ then $a \in A_{\alpha}$ for some $\alpha \in I$. But $A_{\alpha_{0}} \cdot a$ is a left ideal and $A_{\alpha_{0}} \cdot a \subset A_{\alpha}$. Since $A_{\alpha}$ is a minimal left ideal (as a left ideal and group) $A_{\alpha_{0}} \cdot a=A_{\alpha}$. Now

$$
\left[\frac{1}{N} \sum_{i=1}^{N} l_{a_{i}} f\right](a)=\frac{1}{N} \sum_{i=1}^{N} f\left(a_{i} a\right)=\frac{1}{N} \sum_{g \in A_{\alpha}} f(g)=(T f)(\alpha) .
$$

But by assumption $(T f)(\alpha)=0$ for each $\alpha \in I$, which implies that

$$
\left[\frac{1}{N} \sum_{i=1}^{N} l_{a_{i}} f\right](g)=0 \quad \text { for each } g \text { of } A .
$$

But since $\phi$ is left invariant

$$
\phi(f)=\phi\left[\frac{1}{N} \sum_{i=1}^{N} l_{a_{i}} f\right]=\phi\left[\left(\frac{1}{N} \sum_{i=1}^{N} l_{a_{i}} f\right) 1_{A}\right]=\phi(0)=0
$$

which proves that if $T f=0$ for some $f$ of $m(G)$ then $\phi(f)=0$ for each $\phi$ of $\operatorname{gl}(G)$.

Let now $\phi_{0}$ be an arbitrary but fixed element of $g l(G)$. We define $\psi_{0}$ of $m(I)^{*}$ such that $T^{*} \psi_{0}=\phi_{0}$ as follows: if $h$ is in $m(I)$ then let $f \in m(G)$ be such that $T f=h$ (by (5) above there exists such an $f$ ). We define $\psi_{0}(h)=\phi_{0}(f)$. $\psi_{0}$ is well defined on $m(I)$, since if $f_{1}$ is such that $T f_{1}=h=T f$ then $T\left(f_{1}-f\right)=0$ and thus $\phi_{0}\left(f_{1}-f\right)=0$. We get that $\phi_{0}\left(f_{1}\right)=\phi_{0}(f)=\psi_{0}(h)$.

$\psi_{0}$ is linear since if $h_{i}=T f_{i}, i=1,2$, then $T\left(\alpha f_{1}+\beta f_{2}\right)=\alpha T f_{1}+\beta T f_{2}$ and $\psi_{0}\left(\alpha h_{1}+\beta h_{2}\right)=\phi_{0}\left(\alpha f_{1}+\beta f_{2}\right)=\alpha \phi_{0}\left(f_{1}\right)+\beta \phi_{0}\left(f_{2}\right)=\alpha \psi_{0}\left(h_{1}\right)+\beta \psi_{0}\left(h_{2}\right)$.

If $h$ is in $m(I)$ then we can choose by (5) above a $f$ in $m(G)$ such that $\|f\| \leqq\|h\|$ and $T f=h$. Thus $\left|\psi_{0}(h)\right|=\left|\phi_{0}(f)\right| \leqq\left\|\phi_{0}\right\|\|f\| \leqq\left\|\phi_{0}\right\|\|h\|$ which implies that $\psi_{0}$ is in $m(I)^{*}$. But for $f$ in $m(G)$ let $h=T f$, then $\left(T^{*} \psi_{0}\right)(f)=\psi_{0}(T f)=\psi_{0}(h)=\phi_{0}(f)$ which proves that $T^{*} \psi_{0}=\phi_{0}$, in other words that $T^{*}$ is a linear isometry from $m(I)^{*}$ onto $g l(G)$.

If now $\psi \in m(I)^{*}$ is non-negative (i.e., $\psi(h) \geqq 0$ for $h \geqq 0$ ) and if 
$f \in m(G)$ is non-negative then by (1) above $T f$ is non-negative and $\left(T^{*} \psi\right)(f)=\psi(T f) \geqq 0$ which proves that $T^{*}$ is positive.

If $\psi \in c_{0}(I)^{\perp}$ (i.e., $\psi\left(1_{\alpha}\right)=0$ for each $\left.\alpha \in I\right)$ and if $B \subset G$ is a finite set then $\left(T^{*} \psi\right)\left(1_{B}\right)=\psi\left(T 1_{B}\right)=0$, since by $(6)$ above $T\left(1_{B}\right)$ does not vanish at most on a finite set. Thus $T^{*} \psi$ is in $c_{0}(G)^{\perp}$ and $T^{*}\left(c_{0}(I)^{\perp}\right) \subset c_{0}(G)^{\perp}$ $\cap \operatorname{sl}(G)$.

If now, $\psi$ is in $Q l_{1}(I)\left(\|\psi\|=\sum_{\alpha \in I}\left|\psi\left(1_{\alpha}\right)\right|<\infty\right)$ then we define $\phi$ in $Q l_{1}(G)$ as follows:

$\phi\left(1_{o}\right)=\psi\left(1_{\alpha}\right)$ for $g \in A_{\alpha}, \alpha \in I$ and $\phi\left(1_{o}\right)=0$ for $g \in G-A$ (if nonvoid). $\psi\left(1_{\alpha}\right) \neq 0$ at most on a countable subset of $I$, therefore $\phi\left(1_{o}\right) \neq 0$ at most on a countable subset of $G$ and

$$
\sum_{o \in G}\left|\phi\left(1_{o}\right)\right|=\sum_{\alpha \in I} \sum_{o \in A_{\alpha}}\left|\phi\left(1_{\sigma}\right)\right|=\sum_{\alpha \in I} N\left|\psi\left(1_{\alpha}\right)\right|=N\|\psi\|<\infty .
$$

Moreover,

$$
\begin{aligned}
\left(T^{*} \psi\right)(f) & =\psi(T f)=\sum_{\alpha \in I} \psi\left(1_{\alpha}\right)(T f)(\alpha)=\sum_{\alpha \in I} \psi\left(1_{\alpha}\right) \frac{1}{N} \sum_{\sigma \in A_{\alpha}} f(g) \\
& =\sum_{o \in G} \frac{1}{N} \phi\left(1_{o}\right) f(g)
\end{aligned}
$$

which implies that $T^{*} \psi=(1 / N) \phi$ and $\phi \in Q l_{1}(G)$. Thus $T^{*}\left(Q l_{1}(I)\right)$ $C Q l_{1}(G) \cap g l(G)$.

If $\phi \in c_{0}(G)^{\perp} \cap \operatorname{gl}(G)$ then there is some $\psi \in m(I)^{*}$ such that $T^{*} \psi=\phi$. However as well known, $c_{0}(I)^{\perp} \oplus Q l_{1}(I)=m(I)^{*}$ (see [7, p. 429]), which implies that $\psi$ can be decomposed into $\psi=\psi_{1}+\psi_{2}$ with $\psi_{1} \in Q l_{1}(I)$ and $\psi_{2} \in c_{0}(I)^{\perp}$. Thus $T^{*} \psi=T^{*} \psi_{1}+T^{*} \psi_{2}=\phi$. But from above, $T^{*} \psi_{2} \in c_{0}(G)^{\perp}$ and by assumption $\phi \in c_{0}(G)^{\perp}$, which implies that $T^{*} \psi_{1} \in c_{0}(G)^{\perp} \cap Q l_{1}(G)=\{0\}$.

We have shown that $T^{*}\left(c_{0}(I)^{\perp}\right)=c_{0}(G)^{\perp} \cap g l(G)$. In the same way one gets that $T^{*}\left(Q l_{1}(I)\right)=Q l_{1}(G) \cap g l(G)$ which finishes the proof of the theorem.

ReMARKs. If $\phi_{1}, \phi_{2} \in m(I)^{*}$ and $x \in m(I)$ then $\left(\phi_{1} \odot \phi_{2}\right)(x)=\phi_{1}\left(\phi_{2} l_{\alpha}^{\prime} x\right)$ where $l_{\alpha}^{\prime}$ is the left translation operator in $m(I)$ with respect to the element $\alpha \in I$. (See M. M. Day [3, p. 527].) Since $\left(l_{\alpha}^{\prime} x\right)(\beta)=x(\alpha \beta)$ $=x(\beta)$ one gets that $\left(\phi_{1} \odot \phi_{2}\right)(x)=\phi_{1}\left(\phi_{2}(x) 1_{I}\right)=\phi_{1}\left(1_{I}\right) \cdot \phi_{2}(x)$ and thus $\phi_{1} \odot \phi_{2}=\phi_{1}\left(1_{I}\right) \phi_{2}$, which implies that $T^{*}\left(\phi_{1} \odot \phi_{2}\right)=\phi_{1}\left(1_{I}\right) T^{*} \phi_{2}$. (Until now $\odot$ denoted multiplication in $m(I)^{*}$. From now on its denotes multiplication in $m(G)^{*}$.) But $\left(T^{*} \phi_{1}\right) \odot\left(T^{*} \phi_{2}\right)=\left(\left(T^{*} \phi_{1}\right)\left(1_{G}\right)\right) T^{*} \phi_{2}$ (see Day [3, p. 530]). Since $\left(T^{*} \phi_{1}\right)\left(1_{G}\right)=\phi_{1}\left(T 1_{G}\right)=\phi_{1}\left(1_{I}\right)$ one gets that $T^{*}\left(\phi_{1} \odot \phi_{2}\right)=\left(T^{*} \phi_{1}\right) \odot\left(T^{*} \phi_{2}\right)$ which implies the

CoRollary. $T^{*}$ is an algebraic isomorphism from $m(I)^{*}$ onto $g l(G)$. 
Remarks. If $\phi \in \operatorname{gl}(G)$ then let $\psi=\left(T^{*}\right)^{-1}(\phi)$. By Jordan's decomposition theorem $\psi=\psi_{1}-\psi_{2}$ with non-negative $\psi_{1}, \psi_{2}$ of $m(I)^{*}$ (see $\left[4\right.$, p. 98]). Thus $\phi=T^{*} \psi=T^{*} \psi_{1}-T^{*} \psi_{2}$ where $T^{*} \psi_{i} i=1,2$ are nonnegative disjoint left invariant elements in $m(G)^{*}$. If $\alpha_{i}=\left(T^{*} \psi_{i}\right)\left(1_{G}\right)$ $\geqq 0$ then it is easily seen that $\phi$ can be written as $\phi=\alpha_{1} \phi_{1}-\alpha_{2} \phi_{2}$ where $\phi_{1}, \phi_{2}$ are either left invariant means or zero. $\left(\phi_{i}=\left(1 / \alpha_{i}\right) T^{*} \psi_{i}\right.$ if $\alpha_{i}>0$ and $\phi_{i}=0$ if $\alpha_{i}=0$.) For semigroups with cancellation, this is a result of M. M. Day [2, p. 281].

\section{REFERENCES}

1. A. H. Clifford, Semigroups containing minimal left ideals, Amer. J. Math. 70 (1948), 521-526.

2. M. M. Day, Means for the bounded functions and ergodicity of the bounded representations of semigroups, Trans. Amer. Math. Soc. 69 (1950), 276-291.

3. - A Amenable semigroups, Illinois J. Math. 1 (1957), 509-544.

4. N. Dunford and J. Schwartz, Linear operators. I, Interscience, N. Y., 1958.

5. E. Granirer, On amenable semigroups with a finite dimensional set of invariant means. I, Illinois J. Math. (to appear).

6. - On amenable semigroups with a finite dimensional set of invariant means. II, Illinois J. Math. (to appear).

7. G. Köthe, Topologische lineare Räume, Springer, Berlin, 1960.

The Hebrew University of Jerusalem 